\title{
Design \& Control of Magnetic Levitation System ED-4810: Review and Stability Test
}

\author{
Muhammad Junaid Khan*, Daud Khan, Sadia Jabeen Siddiqi, Salman Saleem, Imran Khan
}

Department of Electrical Engineering, University of Engineering \& Technology, Pakistan

Received July 20, 2019; Revised August 22, 2019; Accepted September 1, 2019

\begin{abstract}
Copyright $\odot 2019$ by authors, all rights reserved. Authors agree that this article remains permanently open access under
\end{abstract} the terms of the Creative Commons Attribution License 4.0 International License

\begin{abstract}
This manuscript deals with the simulation and comparison of linear as well as non-linear results for Magnetic Levitation System ED-4810 for different parameters assigned to the model. At first, different results for the simulations are achieved, using Simulink R2017a as a tool. Different results achieved through simulations were than analyzed. Magnetic Levitation System considered in the manuscript, taken as a steel ball suspended in a voltage controlled magnetic field. Furthermore, state space controller is modeled in order to gain the linearity of the system. This study demonstrates the modeling, simulations and comparison of the model ED-4810 upon which, Routh Hurwitz Criterion is executed to accomplish the stability test of the system.
\end{abstract}

Keywords Magnetic Levitation System ED-4810, Simulink R2017a, Routh Hurwitz Criterion, State Space Controller

This paper is an extension of work originally presented in 2018 IEEE 21st International Multi-Topic Conference (INMIC).

\section{Introduction}

Magnetic levitation system (MLS) is known as electro-mechanical systems in which an object is levitate in specific region without using any support. Now a days this technology is spreading over a wide range because of its contact-less and friction-less properties as it removes energy losses which occurs due to friction [3]. In various fields it has been used over a wide range such as highly accurate positioning systems [4], bearing less motors [5] [6], magnetic levitation vehicles [7] and wind turbines [8], micro robot actuation [9], and biomedical devices [10]. Since this technology develops, researchers have analyzed a large number of such systems. In the analysis of such systems most of the work has been done on designing of controller as they require appropriate control action and are more complex, such as State space controller [11], feedback linearization is used to control the position of ball [12]. However, there are mostly highly non-linear systems and open loop unstable. This nonlinearity and un-stability feature of magnetic levitation systems makes their control and modelling very challenging [4].

The Magnetic Levitation system considered in the manuscript is made-up of a steel ball levitated in a voltage-controlled magnetic field [11]. The system we are using for our analysis is the educational nonlinear hardware model of Magnetic Levitation System ED-4810. During the study of the system, we attain different simulation by using different physical parameters for the model of magnetic levitation system ED-4810. Result produced were than analyzed and compare with the result of the model of MLS [1]. For the purpose of linearization state space is implemented to gain the linear position of the system, after than different results for linear system is compared with the result of linear MLS [1].

State space equations are models that practice state variables by a set of first order differential equations to define a scheme in the time-domain. This eradicates the necessity of executing the profound Laplace transform and partial fraction expansions to examine a control system. Henceforth, state space illustration is a mathematical model of a system as a set of inputs, outputs \& state variables related by first order differential equation.

Routh-Hurwitz stability criterion is a mathematical test that is an essential and adequate condition for the stability of a linear time invariant (LTI) control systems. In this paper, Routh Hurwitz stability test is executed to examine the stability condition of the system. Initially, Eigen values are used to produce equations required for transfer function, and then Routh Locus result is generated in the MATLAB. Finally, first column co-efficient in the Routh Hurwitz array are examined in order to get final result about the stable condition of the system. This criterion is also to check the stability of the model. [1]

Concerning this paper, Section-3 presents the general description of a magnetic levitation system and its various components. Section-4 represents the modelling of the magnetic system ED-4810. Section-5 represents simulation 
and results for non-linear system. Moreover, linearization of the system is discussed in Section-6 of the paper and Section-7 presents explanation about the stability test. Finally, conclusion of the entire work is given in Section-8 of the paper.

\section{Magnetic Levitation System}

ED-4810 Magnetic Levitation System is an experimental unit allowing theoretical verification of the principle of magnetic levitation by emerging steel ball in the air with magnetic force, and this unit is manufactures suitably for experimental education on modelling and control theory of physical system as the one using the principle of position sensor and feedback control of steel ball for which magnetic force and optical sensor are used. Fig.1. shows the diagram of the system.

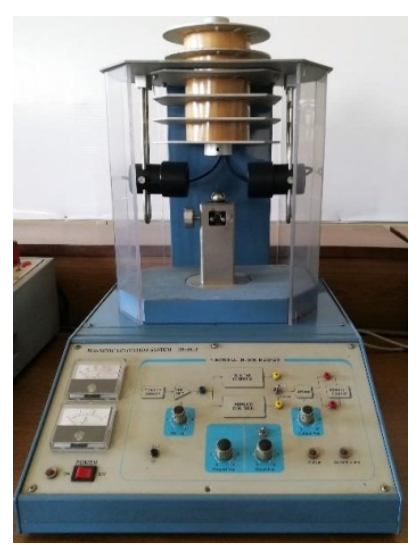

Figure 1. Magnetic Levitation Model ED-4810 [1]

\section{Modelling of Magnetic Levitation System (ED-4810)}

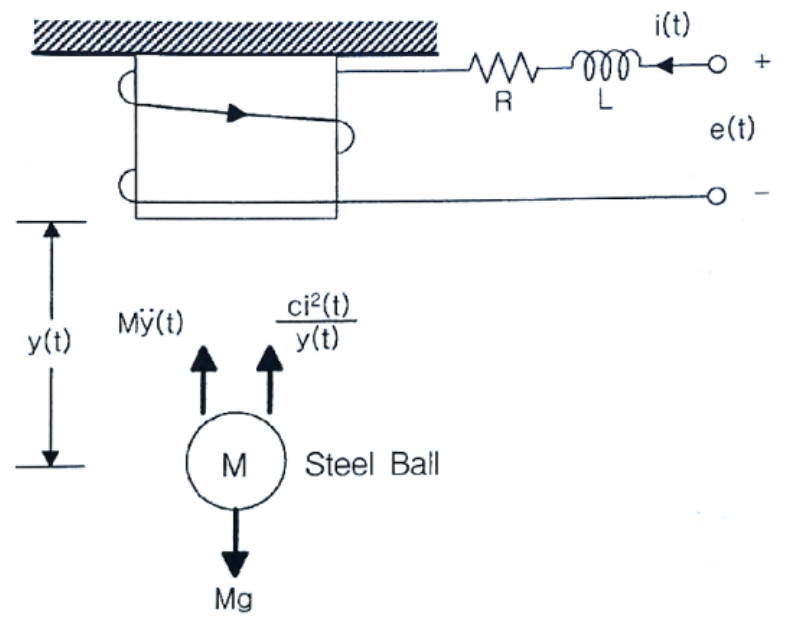

Figure 2. Model of Magnetic Levitation System

The Magnetic Levitation System may be modeling into the derivative of equation of electric current composing an electromagnet and the equation of motion of steel ball produced by the motion of steel ball. Fig. 2 shows a sample model of magnetic levitation system.

As above model of MLS, suppose it is suitably modelling the electromagnet coil by an assembly of resistor and inducer as the inductance $L$ in the coil and resistance $R$ is connected directly to the external of source applied. Where,

$\boldsymbol{M}$ is weight of steel ball

$\mathbf{Y}(\boldsymbol{t})$ is the center position of the ball

$\boldsymbol{C}$ is fixed positive number (gain)

$\mathbf{i}(\boldsymbol{t})$ is electric current flowing on an electromagnetic coil

$\boldsymbol{L}$ is the total inductance and

$\boldsymbol{e}(\boldsymbol{t})$ is the source applied.

The notable variables are distance between ball and electromagnet and current flowing in electromagnetic coil. The total inductance provided is as;

$$
L=L_{1}+\frac{L_{0} y_{0}}{y(t)}
$$

The relation between voltage and electric current in the coil in Fig. 2. Can be represented as;

$$
e(t)=R i(t)+L \frac{d i(t)}{y(t)}
$$

The equation of motion for a ball in induced from the relation between all forces acted on the ball and acceleration of the ball as;

$$
M \frac{d^{2} y(t)}{d t^{2}}=M g-\frac{c i^{2}(t)}{y(t)}
$$

Where, if the condition variable number is

$x_{a}(t)=y(t), x_{b}(t)=y(t), x_{c}(t)=i(t)$ and input $u(t)=e(t)$, the derivative equation is as follow;

$$
\begin{gathered}
\dot{x_{a}}(t)=x_{2}(t) \\
\dot{x_{b}}(t)=g-\frac{c}{M} \frac{x_{c}^{2}(t)}{x_{a}(t)} \\
\dot{x_{c}}(t)=-\frac{R}{L} x_{c}(t)+\frac{1}{L} u(t)
\end{gathered}
$$

\subsection{Unstable Equilibrium Point of System}

The purpose of the magnetic levitation experimental unit is to let the steel ball keep emerging in the air by controlling the strength of the magnet. In the most conditions, simply increase in the current of electromagnet to be equal to gravity. As Fig. 2, the steel ball is staying in the air temporarily by the equilibrium of power at the point where the magnetic force of electromagnet and the gravity are same but the equilibrium isn't available to keep and is broken soon. Because of the distance between an electromagnet and steel ball is kept away with constant electric current, that is, if $\mathrm{y}(\mathrm{t})$ becomes large, the steel ball is dropped by weaken power than an electromagnet pulls the steel ball. And of the distance is closed, that is, if $y(t)$ becomes small, the steel ball is attached on the electromagnet by increased power. In the system theory, 
the point indicating the unstable equilibrium status is called the Unstable Equilibrium Point.

Let suppose $\frac{d^{2} y(t)}{d t^{2}}=0$, and the gravity acting on the steel ball is constant as $M g$ without being changed proportion to the weight of the steel ball, thus if the distance spaced from the center of the ball is $y^{*}$ and an electric current required for an equilibrium between the gravity and magnetic force is $i^{*}$, from the eq. (3), $i^{*}$ should be agreed with

$$
M g=\frac{c i^{* 2}}{y^{*}}
$$

Where, the fixed number c is;

$$
c=\frac{M g y^{*}}{i^{2}}
$$

If the direct resistance of an electromagnet is $\mathrm{R}$ and the voltage available to supply an electric current of $i^{*}$ is $e^{*}$, their relation is $i^{*}=e^{*} / R$, thus

$$
e^{*}=\sqrt{\frac{M g y^{*} R^{2}}{c}}
$$

This is, the impressed voltage of electromagnet $e^{*}$ that a steel ball is located at the point kept away from an electromagnet by $y^{*}$ is a function of $y^{*}$ in eq. (4).

Keep stably emerging the steel ball by overcoming the unstable equilibrium status in the condition that the steel ball is far from an electromagnet by constant distance. Fig.3 shows the feedback control of MLS must be configured.

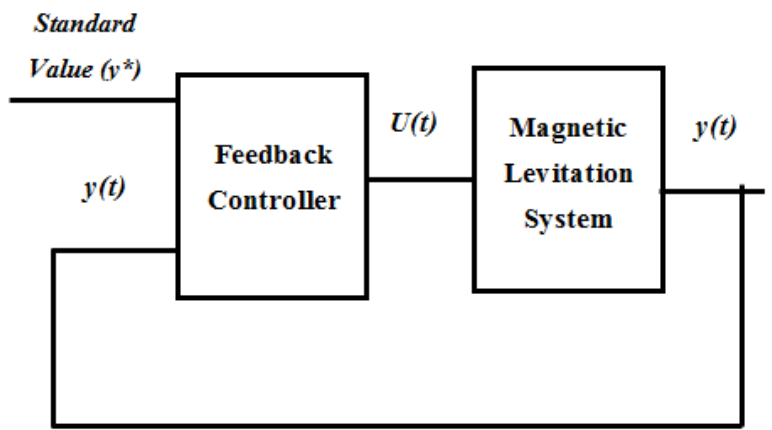

Figure 3. Feedback Control of Magnetic Levitation System

The standard value in the above figure can be just a distance $y^{*}$ that want to keep the center pf steel ball from electromagnet, then the output is an actual distance between the electromagnet and center of the ball $y(t)$. The feedback controller does the function to make an input signal for controlling the strength of an electromagnet by the distance you want $y^{*}$ and actual distance $y(t)$. Then the input of system $\mathrm{u}(\mathrm{t})$ is a permitted voltage of an electromagnet. There is some excessive status by an inductance of an electromagnet, but in the normal condition, the strength of an electromagnet is controlled by being flowed an electric current on electromagnet in proportion to the voltage.

The physical parameters of the system are as shown below. New values are assigned to the system i.e. Mass, Resistance and inductance, while center and current of ball remain the same.

The value of $c$ (which is a fixed number) can be calculated from the eq. (7) as;

$$
c=M g \frac{y^{*}}{i^{* 2}}
$$

Here we will assign two different values to the system in order to differentiate between the results and compare it with the results achieved through physical parameters in Magnetic Levitation Model [1].

Table 1. NEW PARAMETERS ASSIGNED: Having greater values than the model in [1]

\begin{tabular}{|c|c|c|}
\hline Parameters & Values & Units \\
\hline M & 2 & $\mathrm{Kg}$ \\
\hline $\mathrm{R}$ & 50 & $\mathrm{Ohm}(\Omega)$ \\
\hline $\mathrm{L}$ & 0.2 & $\mathrm{H}$ \\
\hline $\mathrm{g}$ & 9.8 & $\mathrm{~m} / \mathrm{sec}^{2}$ \\
\hline $\mathrm{c}$ & 0.3 & -- \\
\hline $\mathrm{y}^{*}$ & 0.03 & $\mathrm{M}$ \\
\hline $\mathrm{i}^{*}$ & 1.44 & $\mathrm{~A}$ \\
\hline
\end{tabular}

Table 2. NEW PARAMETERS ASSIGNED: Having less values than the model in [1]

\begin{tabular}{|c|c|c|}
\hline Parameters & Values & Units \\
\hline M & 0.2 & $\mathrm{Kg}$ \\
\hline $\mathrm{R}$ & 10 & $\mathrm{Ohm}(\Omega)$ \\
\hline $\mathrm{L}$ & 0.12 & $\mathrm{H}$ \\
\hline $\mathrm{g}$ & 9.8 & $\mathrm{~m} / \mathrm{sec}^{2}$ \\
\hline $\mathrm{c}$ & 0.0283 & -- \\
\hline $\mathrm{y}^{*}$ & 0.03 & $\mathrm{M}$ \\
\hline $\mathrm{i}^{*}$ & 1.44 & $\mathrm{~A}$ \\
\hline
\end{tabular}

Table 3. PHYSICAL PARAMETERS OF MLS ED-4810 [1]

\begin{tabular}{|c|c|c|}
\hline Parameters & Values & Units \\
\hline $\mathrm{m}$ & 0.5 & $\mathrm{~kg}$ \\
\hline $\mathrm{R}$ & 20 & $\mathrm{Ohm}(\Omega)$ \\
\hline $\mathrm{L}$ & 0.158 & $\mathrm{H}$ \\
\hline $\mathrm{g}$ & 9.8 & $\mathrm{~m} / \mathrm{sec}^{2}$ \\
\hline $\mathrm{K}$ & 0.08 & -- \\
\hline $\mathrm{y}$ & 0.03 & $\mathrm{M}$ \\
\hline $\mathrm{I}$ & 1.44 & $\mathrm{~A}$ \\
\hline
\end{tabular}

\section{Simulation \& Results for Non-Linear System}

The simulation of the model can be achieved through the endorsed equations and physical parameter by using MATLAB/ Simulink as a tool. The simulation of the conventional non-linear MLS ED-4810 is shown in Figure. 4 in which Input source provided at the input, on the other hand, it gives speed and non-linear position of the system while Figure. 5 shows the inner assembly of the block named as "MLS ED-4810". 


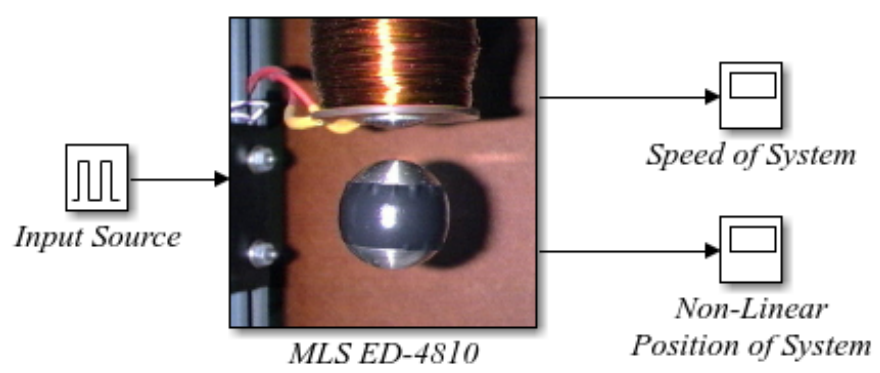

Figure 4. Simulink Model of Non-Linear MLS ED-4810

The interior assembly of the above block "MLS ED-4810" is built-up as;

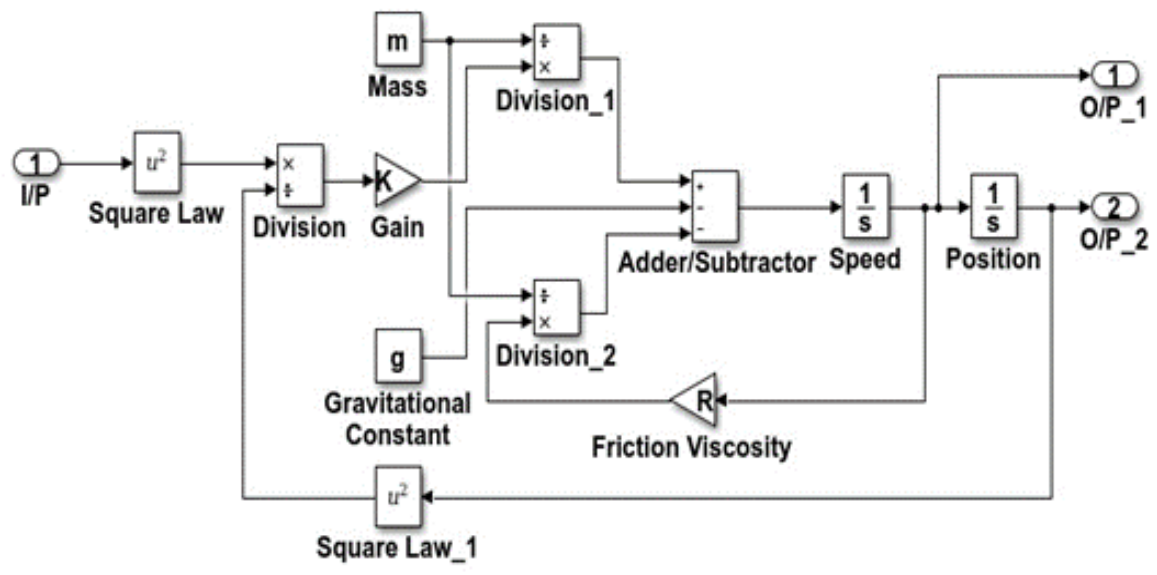

Figure 5. Simulink Model generated for "Magnetic Levitation System"

\subsection{Analysis of Results}

In the graph, it demonstrates that the mean position of the ball increases with increase in mass. Moreover, the amplitude reaches to approximately 1.1 but after 2 bounces of cycle it levitates with constant speed.

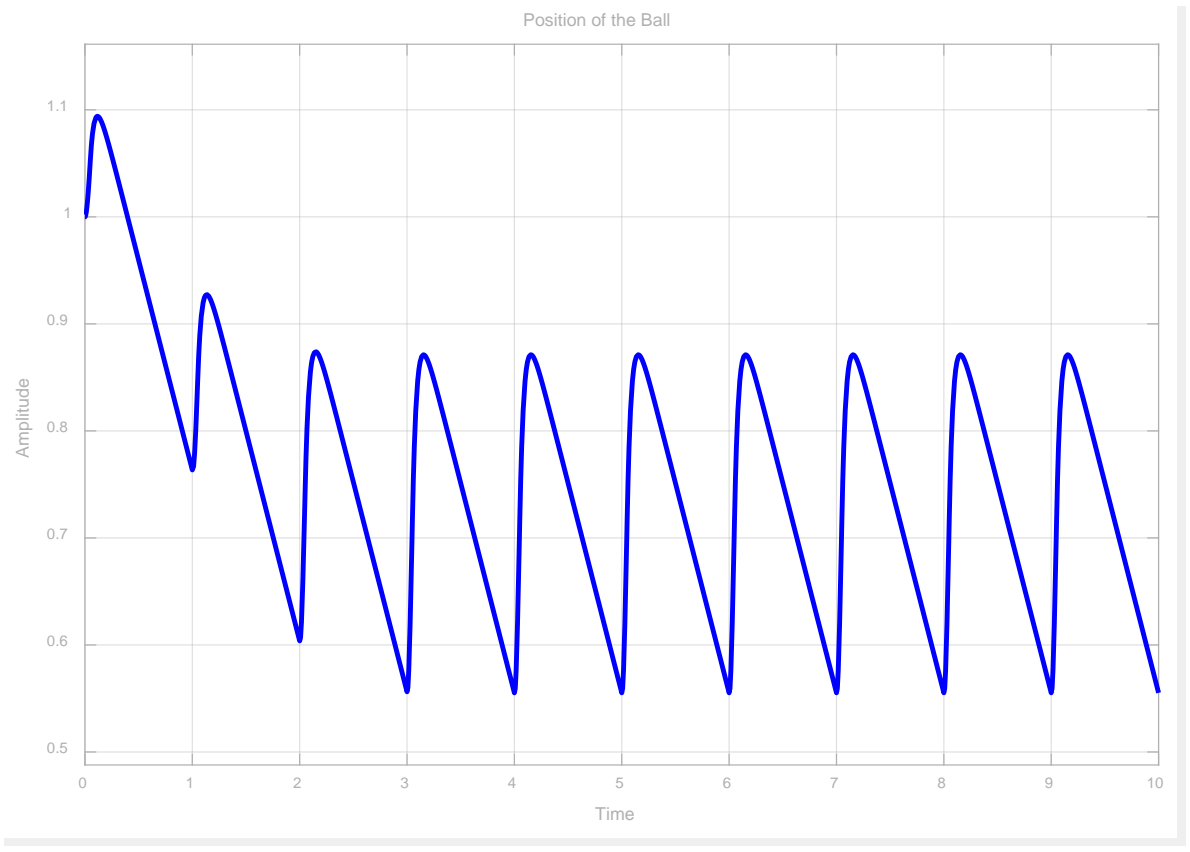

Figure 6. Result of Non-linear Simulation for Table 1 
Similarly, the result for table 2 illustrates that amplitude is decreased but it takes about 5 oscillations which means that with low mass of the ball, it takes more time for constant bouncing.

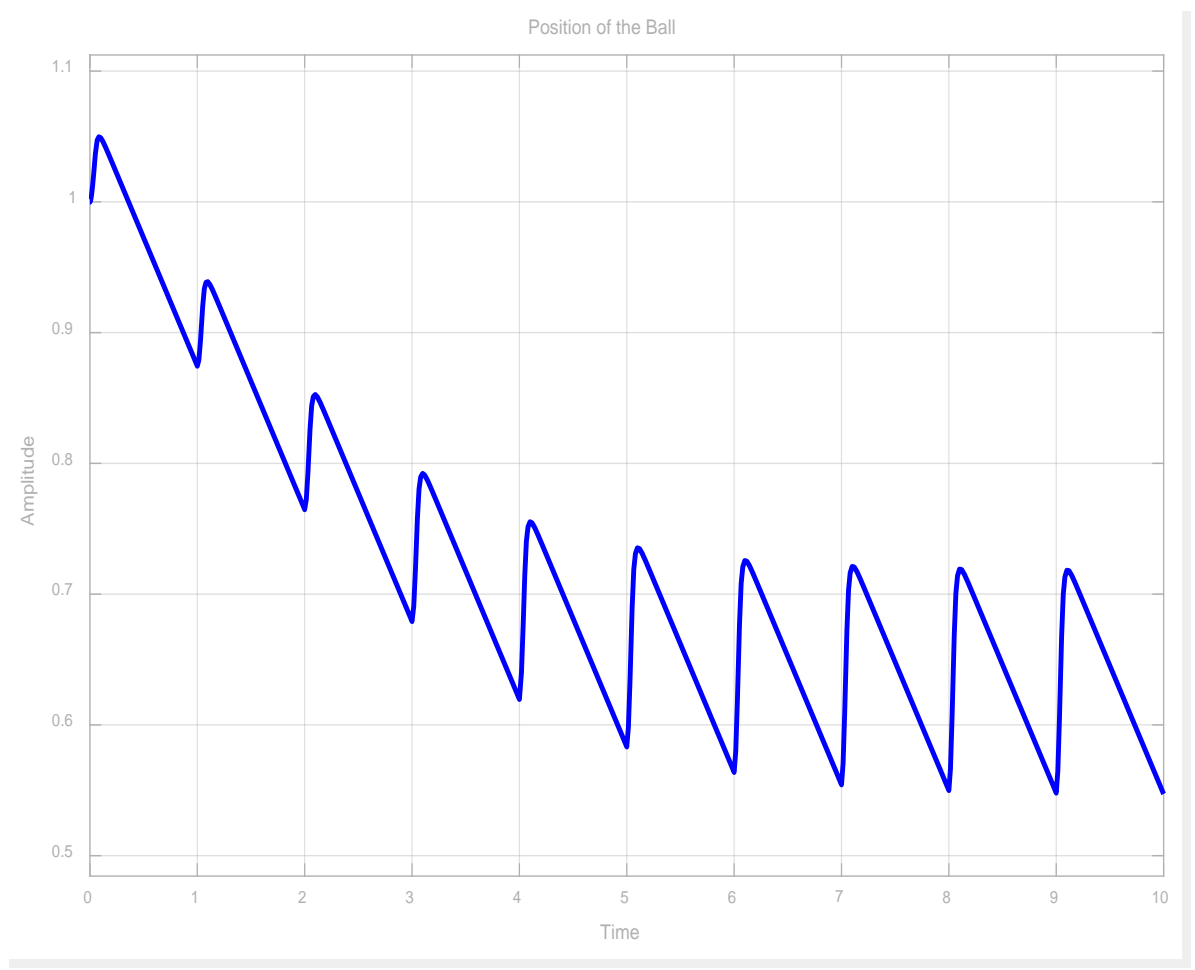

Figure 7. Result of Non-linear Simulation for Table 2

\subsection{Comparison of Results with Model [1]}

By the comparing the resuts of above parameters with model of magentic levitation system [1], one can say that the weight of the mass is directly proportional to amplitude because amplitude increases with increase in mass and inversly proportional to bouncing time of the ball.

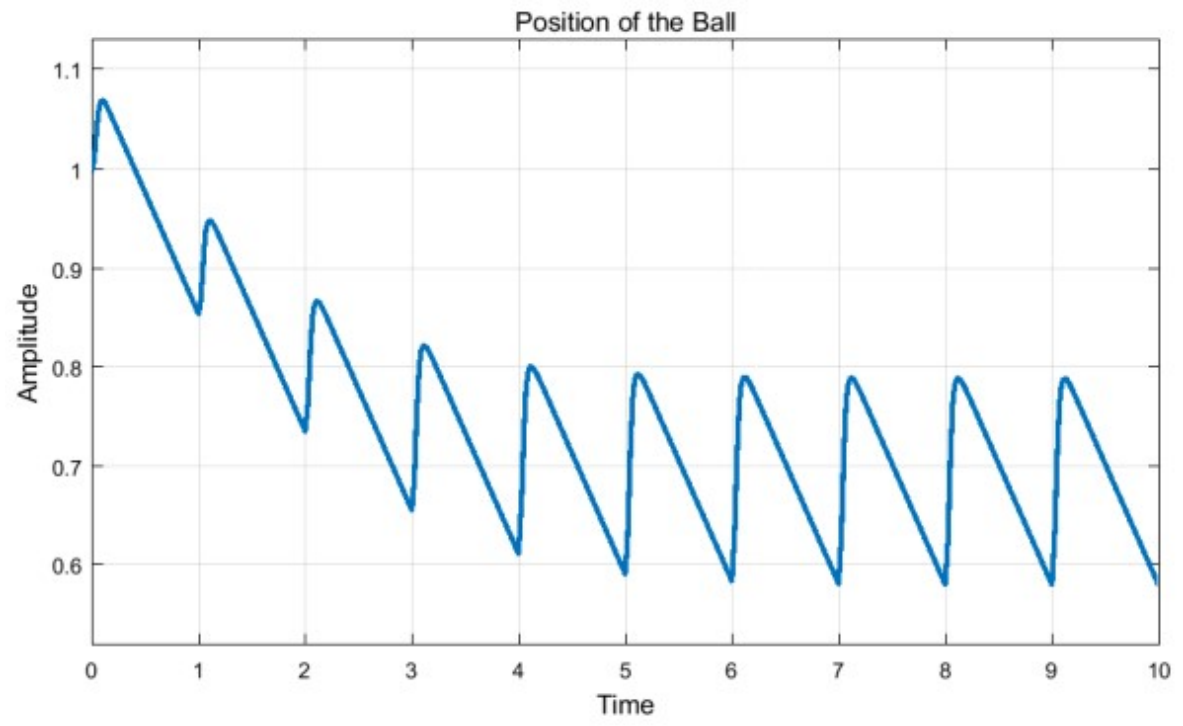

Figure 8. Simulation result of the proposed model [1] 


\section{Linearization of System}

Since the system of Eq. (4) - (6) is non-linear, applying for the method design and analyzation under linear system is not available around the point of motion if linearize the system at one point of motion.

If you want to control to be kept at the point that the steel ball is far from an electromagnet by $y^{*}$, the condition at the point of motion should be;

$$
x_{a}(t)=x_{1}=y^{*}
$$

$x_{1}$ is distance of the ball and $y^{*}$ is defined as its center

$$
x_{b}(t)=x_{2}=0 \text { and } \dot{x_{b}}(t)=0 \text { and } \dot{x_{c}}(t)=0
$$

$x_{2}$ is the time rate of variation of position which must be zero at sturdy state of levitation

Accordingly form (7)-(8);

$$
\dot{x}_{c}(t)=x_{3}=i^{*}=\sqrt{\frac{M g y^{*}}{c}}
$$

$x_{3}$ is the current of the ball at raising time of position.

$$
u(t)=u^{*}=e^{*}=R i^{*}=R \sqrt{\frac{M g y^{*}}{c}}
$$

\subsection{State Space Controller}

If liberalize the non-linear system of Eq. (7) - (9), at these point of motion,

$$
\begin{gathered}
\Delta x(t)=\left[\Delta x_{1}(t) \Delta x_{2}(t) \Delta x_{3}(t)\right]^{\mathrm{T}} \\
\Delta u(t)=u(t)-u^{*}
\end{gathered}
$$

Then, following is the equation of condition liberalized;

$$
\begin{gathered}
\Delta \dot{x}(t)=A \Delta x(t)+B \Delta u(t) \\
\Delta y(t)=C \Delta x(t)
\end{gathered}
$$

Only,

$$
\begin{gathered}
A=\left[\begin{array}{ccc}
0 & 1 & 0 \\
\frac{c x_{3}^{2}}{M x_{1}^{2}} & 0 & -\frac{2 c x_{3}}{M x_{1}} \\
0 & 0 & \frac{-R}{L}
\end{array}\right] \\
\text { Or, } A=\left[\begin{array}{ccc}
0 & 1 & 0 \\
\frac{g}{y^{*}} & 0 & -2 \sqrt{\frac{c g}{M y^{*}}} \\
0 & 0 & \frac{-R}{L}
\end{array}\right] \\
B=\left[\begin{array}{c}
0 \\
0 \\
\frac{1}{L}
\end{array}\right] \\
C=\left[\begin{array}{lll}
1 & 0 & 0
\end{array}\right]
\end{gathered}
$$

From table 1 put the values in Eq. (14) and (15),

$$
A=\left[\begin{array}{ccc}
0 & 1 & 0 \\
326.67 & 0 & -14 \\
0 & 0 & -250
\end{array}\right]
$$

$$
B=\left[\begin{array}{l}
0 \\
0 \\
5
\end{array}\right]
$$

By putting values from table 2 in Eq. (14) and (15), we get;

$$
\begin{gathered}
A=\left[\begin{array}{ccc}
0 & 1 & 0 \\
326.67 & 0 & -13.6 \\
0 & 0 & -83.3
\end{array}\right] \\
B=\left[\begin{array}{c}
0 \\
0 \\
8.33
\end{array}\right]
\end{gathered}
$$

After getting the value of A \& B, these were then added to State Space Parameter Block as show below in Fig. 9 and state space controller is achieved.

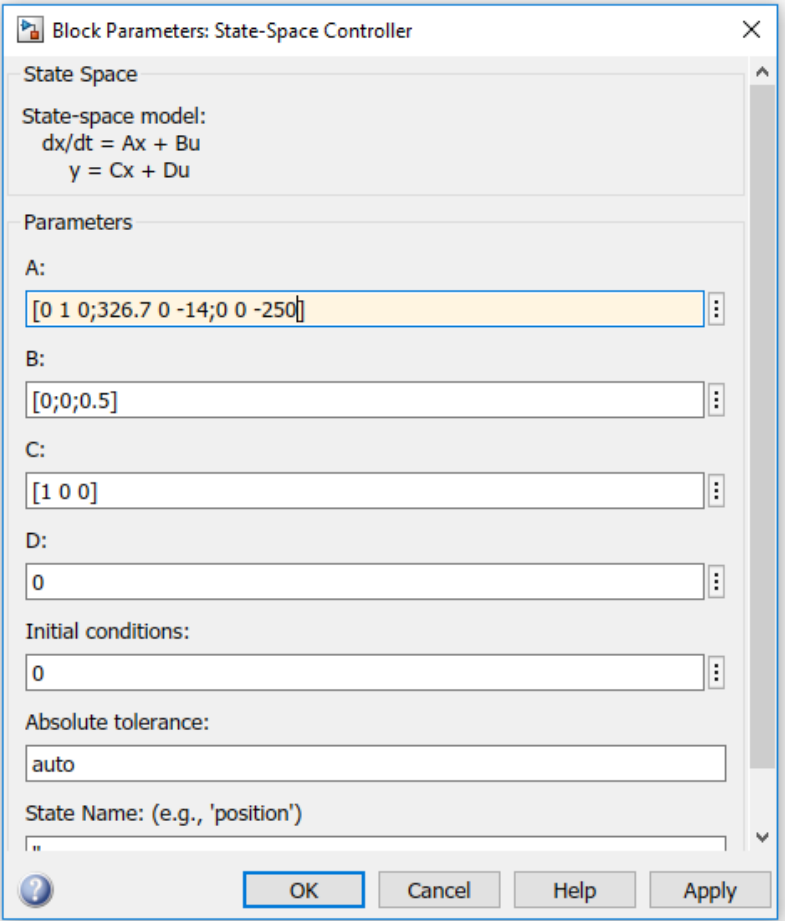

Figure 9. State Space Controller Block Parameter

\subsection{Simulation of the Linear MLS Model}

For the purpose of linearization, state space controller was use from the Simulink library. The controller is connected to the non-linear position of the ball in order to accomplish the linear position.

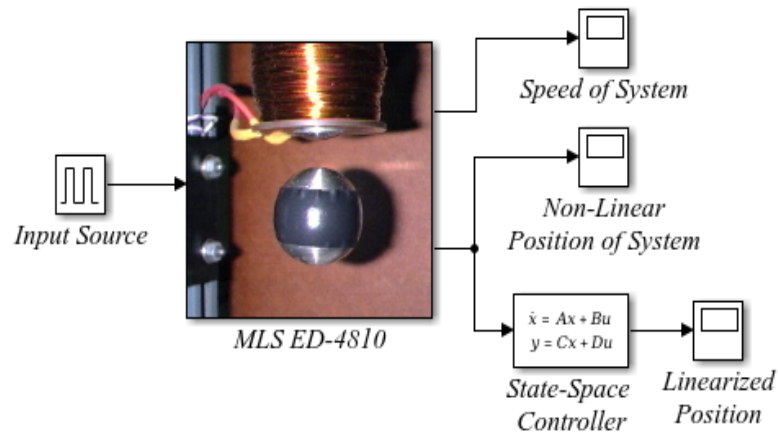

Figure 10. Simulation of the Linear Model of MLS ED-4810 


\subsection{Analysis of Results}

The below graphs depicted that the linearization accuracy varies with respect to change in parameters of the state space controller. Both simulations shows the time period taken is 10 sec but accuracy result of table 1 . simulation is $10^{73}$ while that of table 2 is $10^{75}$.

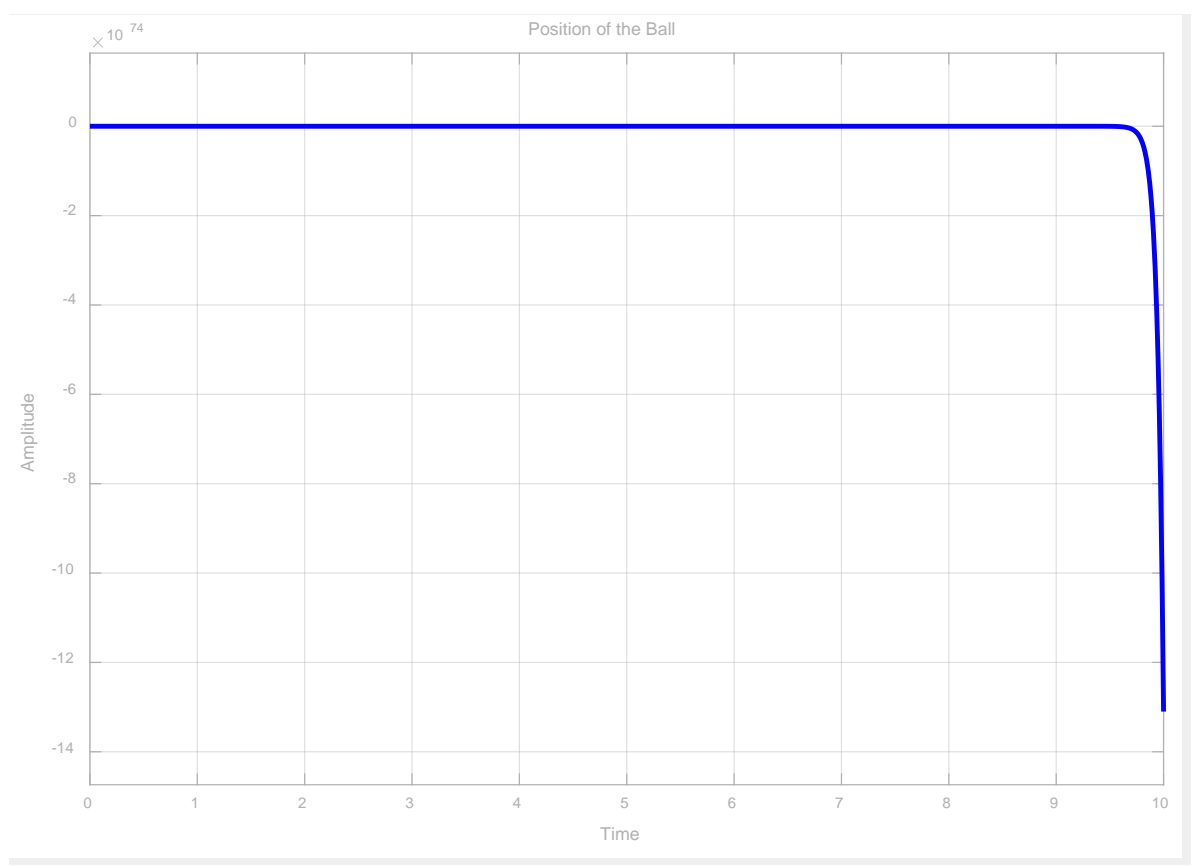

Figure 11. Result of Linear Model simulations for table 1

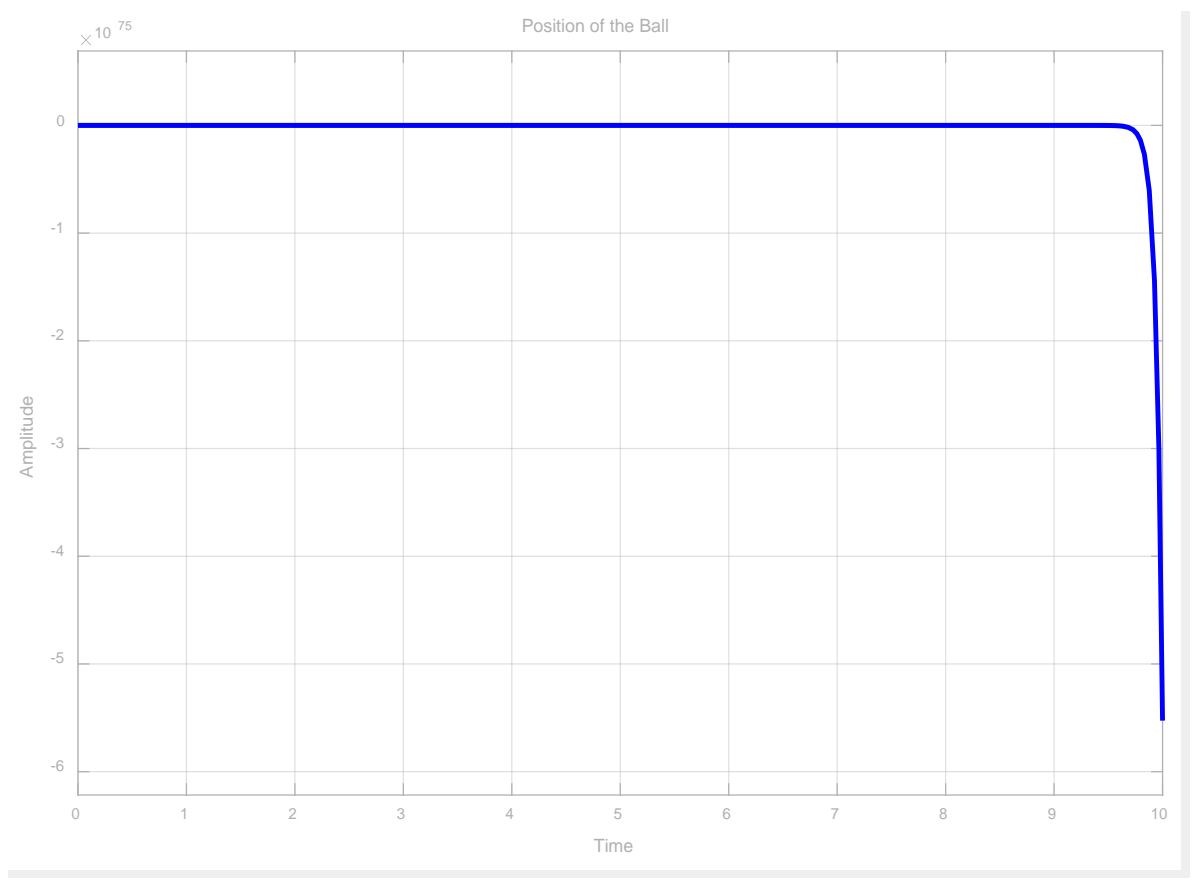

Figure 12. Result of Linear Model simulations for table 2

\subsection{Comparison of Results with Model of MLS [1]}

To relate the results of the above parametrs with the below result of the model in [1], it shows that accuracy of ferromagnetic ball is quiet efficient than a steel ball. The accuracy is $10^{66}$ that of for table. I is $10^{73}$ and table 2 is $10^{75}$ 


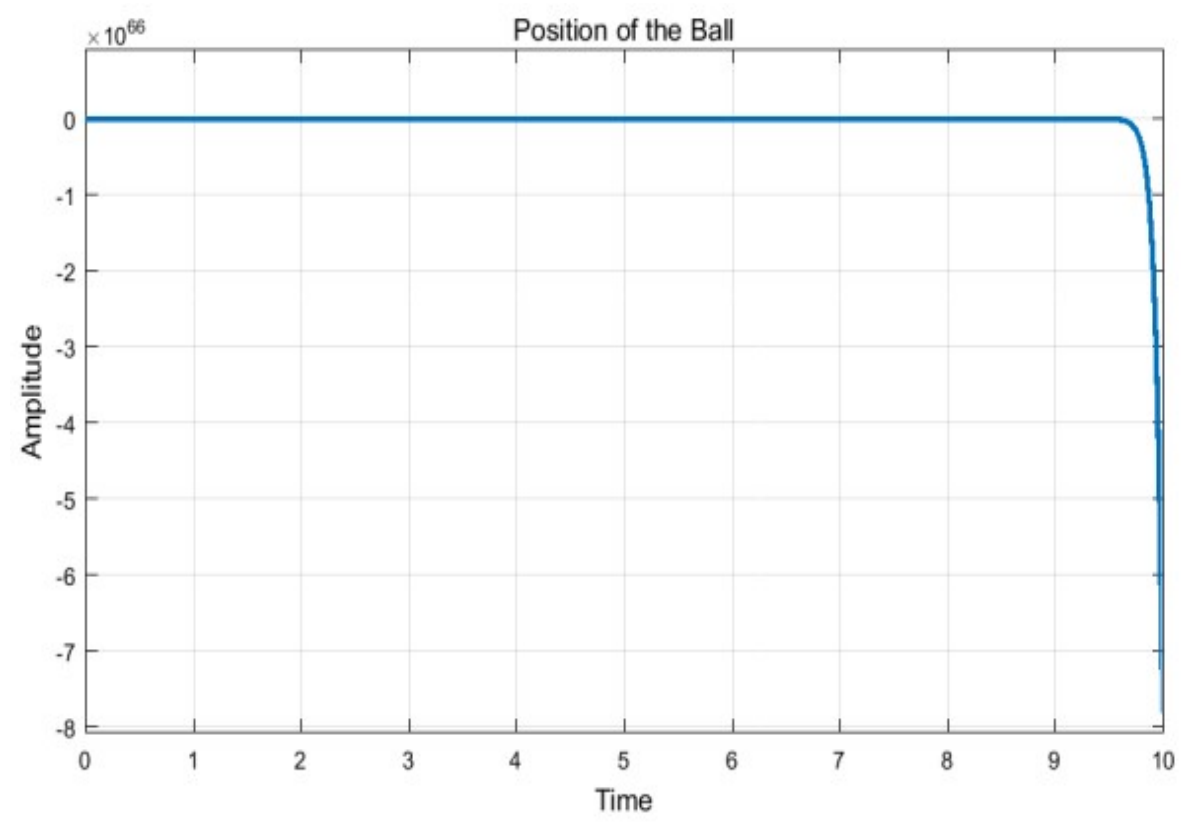

Figure 13. Simulation Result of Linear Model [1]

\section{Stability Test}

For the stability of the system in state space, poles are called as Eigen values.

\subsection{Eigen Values}

Eigen Values are special set of scalars associated with a matrix. They are called characteristic roots, proper values or latent roots.

Since,

$$
\mathrm{G}(\mathrm{S})=\mathrm{C}[\mathrm{SI}-\mathrm{A}]^{-1} \mathrm{~B}+\mathrm{D}
$$

Or,

$$
\lambda=\operatorname{det}([\mathrm{SI}-\mathrm{A}])
$$

We get the equation of the model [1] as,

$$
\mathrm{G}(\mathrm{S})=\mathrm{S}^{3}+316.5 \mathrm{~S}^{2}+334.62 \mathrm{~S}-41360.22
$$

To get the equation of the MLS model ED-4810, put the values in eq. (12), so, equation for the physical parameters of Table 1 is;

$$
G(S)=S^{3}+250 S^{2}-326.67 S-81667.5
$$

And for Table 2 is;

$$
G(S)=S^{3}+833 S^{2}-326.67 S-27211.61
$$

\subsection{Routh Hurwitz Criterion}

A recursive algorithm that helps finding out if a higher order system is stable or not. It is stated as;

"The number of roots of the characteristic polynomial that are in the right hand pole is equal to the number of sign changes in the first column of the Routh Table” [14]

Consider a closed loop Transfer Function,

$G(S)=\frac{b_{0} s^{n}+b_{1} s^{n-1}+\cdots+b_{n-1} s^{1}+b_{n} s^{0}}{a_{0} s^{m}+a_{1} s^{m-1}+\cdots+a_{m-1} s^{1}+a_{m} s^{0}}=\frac{P(S)}{Q(S)}$

Where the co-efficient a \& b are real constant terms and $\mathrm{n} \leq \mathrm{m}$ 


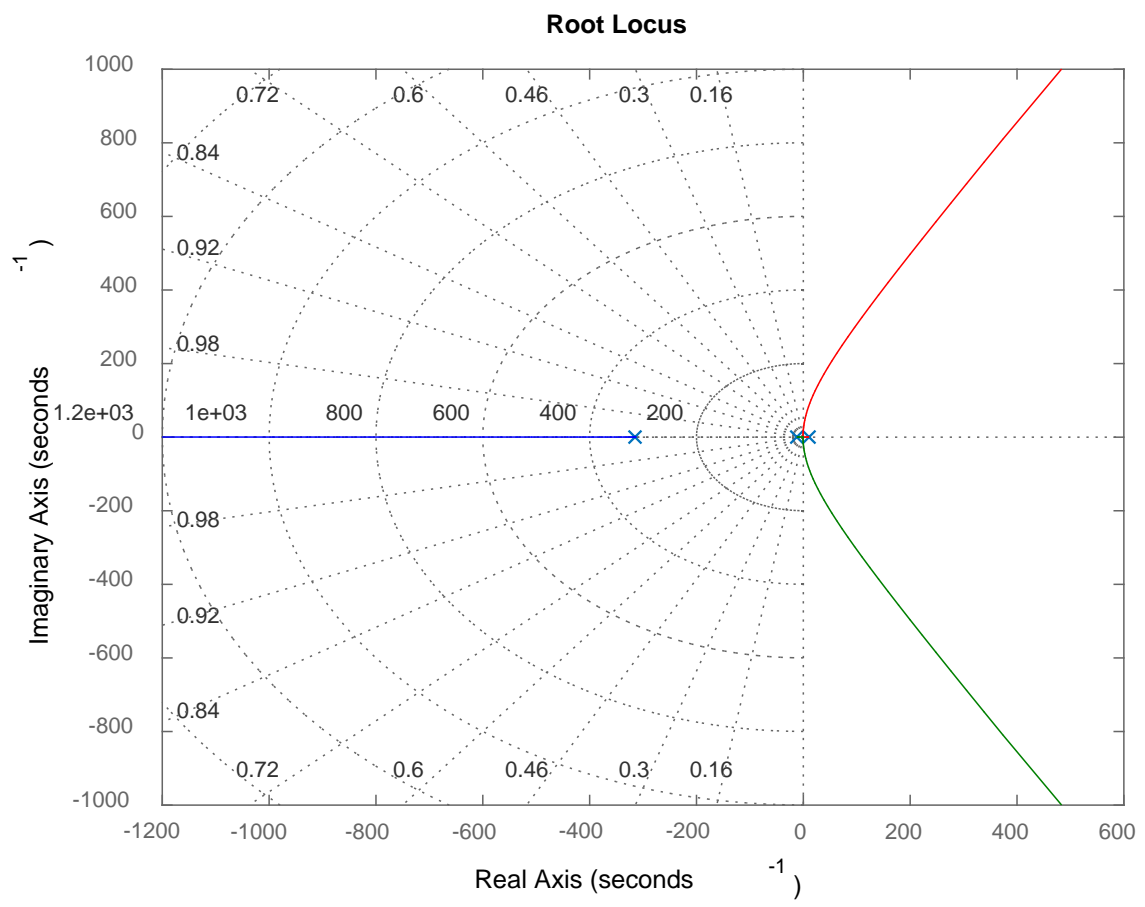

Figure 14. Routh locus resut for MLS model [1].

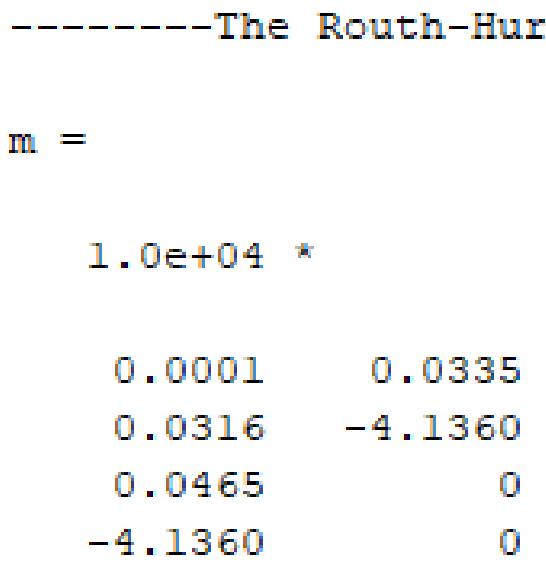

$---->$ System is Unstable <----

Figure 15. Routh Hurwitz array resut for MLS model [1]. 


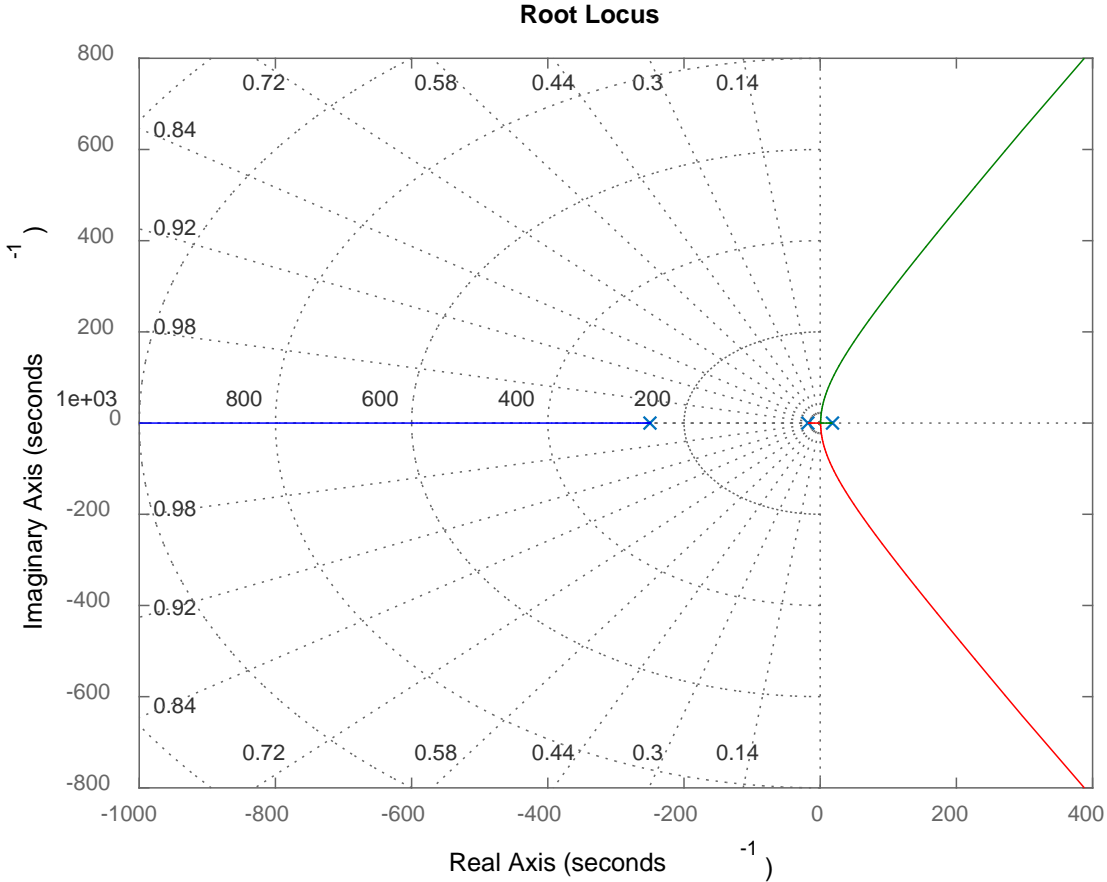

Figure 16. Routh Locus resut for Table. 1 parameters

The Routh-Hurwitz array is:

$\mathrm{m}=$

1. $0 e+04$

$0.0001-0.0327$

$0.0250-8.1668$

$-0.0000$

$-8.1667$

0

$---->$ System is Unstable <----

Figure 17. Routh Hurwitz array resut for Table. 1 parameters 


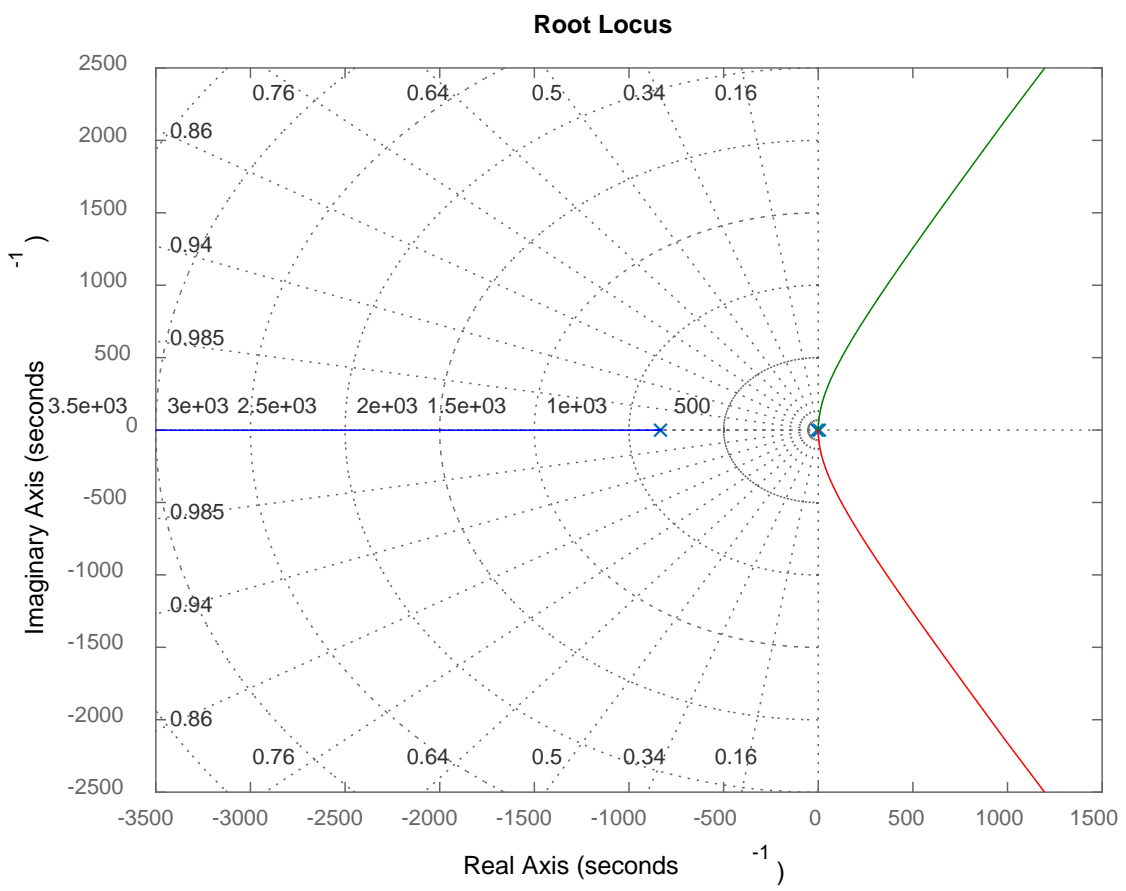

Figure 18. Routh Locus resut for Table. 2 parameters

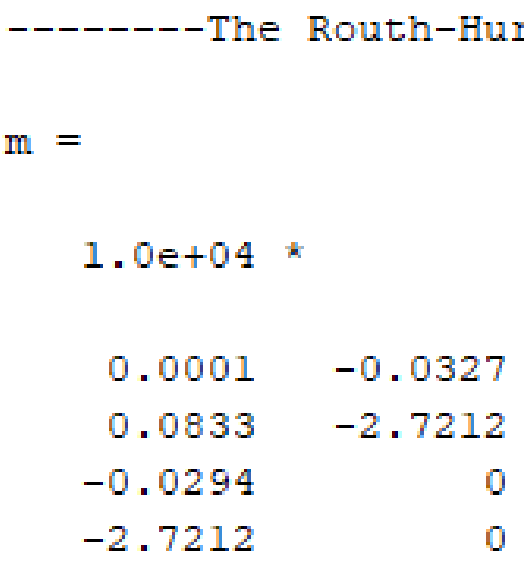

$--->$ System is Unstable <----

Figure 19. Routh Hurwitz array resut for Table. 2 parameters 


\section{Conclusions}

The work presented in this manuscript was grounded on a nonlinear dynamic magnetic levitation system ED-4810 that was examined in terms of its different result for different parameters and compared with the model for linear as well as non-linear simulations. Owing to the non-linear and nature of system, in this work, state space controller is used for linearization of MLS ED-4810 for different parameters. The whole system was demonstrated in vector form and state-equation. Routh Hurwitz Criterion is implemented to get the stability analysis of the system.

\section{REFERENCES}

[1] Muhammad Junaid Khan et.al, "Modelling, Simulation \& Control of Non-Linear Magnetic Levitation System”, IEEE $21^{\text {st }}$ International Multi - Topic Conference (INMIC), 2018.

[2] P. Šuster and A. Jadlovská, "Modeling and Control Design of Magnetic Levitation System”, SAMI 2012, 10th IEEE Jubilee International Symposium on Applied Machine Intelligence and Informatics, January 26-28, 2012 Slovakia.

[3] Tejinder Kumar, Shakshi Rana, S. L. Shimi and Dnyaneshwar Karanjkar, "Modeling, simulation and control of single actuator magnetic levitation system”, Recent Advances in Engineering and computational sciences, UIET Panjab University Chandigarh, pp.1-6, 2014

[4] Petr Chalupa, Martin Maly and Jakub Novak, "Non-Linear Simulink Model of Magnetic Levitation Laboratory Plant”, Proceedings 30th European Conference on Modelling \& Simuliation, Tomas Bata University in Zlin, June 2016.

[5] F. Zurcher, T. Nussbaumer, and J.W. Kolar, "Motor torque and magnetic levitation force generation in bearingless brushless multipole motors," IEEE/ASME Trans. Mechatronics,, vol. 17, no. 6, pp. 1088-1097, Dec 2012.

[6] J. Asama, Y. Hamasaki, T. Oiwa, and A. Chiba, "Proposal and analysis of a novel single-drive bearingless motor," IEEE Trans on industrial electronics, vol. 60, no. 1, pp. 129-138 Jan 2013.

[7] S.M. Jang, Y.P. Park, S.Y. Sung, K.B. Lee, H.W. Cho, and D. J. You, "Dynamic characteristics of a linear induction motor for predicting operating performance of magnetic vehicles based on electromagnetic field theory," IEEE Trasn on magnetics, vol. 47, no. 19, pp. 3673-3676. Oct 2011.

[8] C. V. Aravind, R. Rajparthiban, R. Rajprasad, and Y.V. Wong, "A novel magnetic levitation assisted vertical axis wind turbine-design procedure and analysis,” IEEE 8th International Colloqunium on Signal Processing and its Applications, pp. 93-98, March 2012.

[9] M. Hagiwara, T. Kawahara, T. Iijima, Y. Yamanishi, and F. Arai, "High speed microrobot actuation in amicrofluidic chip by levitated structure with riblet surface," IEEE International Conference on Robotics and Automation, pp. 2517-2522, May 2012.
[10] K. Qian, Z. Xu, and H. Wang, "Investigation on applying passive magnetic bearings to impeller left ventricular assist devices (LVAD)", K. Qian, Z. Xu, and H. Wang, International Conference on Biomedical Engineering and Informatics, pp. 1516-1518, Oct 2010.

[11] Valer Dolga, Lia Dolga, "MODELLING AND SIMULATION OF A MAGNETIC LEVITATION SYSTEM”, Fascicle of Management and Technological Engineering, Volume VI (XVI), 2007.

[12] Rudi Uswarman, Adha Imam Cahyadi, and Oyas Wahyunggoro, "Control of a magnetic levitation system using feedback linearization”, International Conference on Computer, Control, Informatics and Its Applications, Jakarta, Indonesia, pp. 95-98, 2013.

[13] Ishtiaq Ahmad, Muhammad Akram Javaid, "Nonlinear Model \& Controller Design for Magnetic Levitation System", RECENT ADVANCES in Signal Processing, Robotics And Automation, ISBN: 978-960-474-157-1, Feb 2010.

[14] Norman S. Nise, Control System Engineering Book (Fourth Edition), 2010. 